\title{
Tradução
}

\section{Uma tradução da demonstração original do teorema de Bertrand ${ }^{1}$}

\author{
A translation of the original proof \\ of Bertrand's theorem
}

FILADELFO CARDOSO SANTOS

VITORVANI SOARES

ALEXANDRE CARLOS TORT

Instituto de Física da Universidade Federal do Rio de Janeiro | UFRJ

RESUMO Apresenta-se aqui uma tradução da demonstração original de um teorema que se deve a J. Bertrand e que diz respeito às leis de atração que admitem órbitas fechadas e limitadas para condições iniciais arbitrariamente escolhidas.

Palavras-chave mecânica clássica, forças centrais, órbitas.

ABSTRACT A beautiful theorem due to J. L. F. Bertrand concerning the laws of attraction that admit bounded closed orbits for arbitrarily chosen initial conditions is translated from French into Portuguese.

Key words classical mechanics, central forces, orbits.

\section{Introdução}

Em 1873, Joseph Bertrand publicou uma curta, porém importante, memória na qual demonstrava a existência de apenas dois campos centrais para os quais todas as órbitas radialmente limitadas eram fechadas, a saber: a lei newtoniana da gravitação universal, que Bertrand designava como a lei da natureza, e o oscilador harmônico isotrópico. ${ }^{2}$

Considerado uma criança prodígio, Joseph Louis François Bertrand nasceu em 11 de março de 1822 e foi admitido na École Polytechnique aos 17 anos de idade. Fez uma brilhante carreira acadêmica e tornou-se professor titular do Collège de France em 1822, sucedendo a Jean Baptiste Biot como professor de física e matemática. Além de se dedicar à astronomia, Bertrand também trabalhou em teoria dos números, geometria diferencial, teoria das probabilidades e termodinâmica. Ele escreveu vários livros sobre esses temas e, dentre eles, o livro Les fondateurs de l'astronomie moderne: Copernic, Tycho Brahé, Kepler, Gallilée, Newton foi recentemente traduzido para o português. ${ }^{3}$ 
0 teorema de Bertrand relativo às leis de atração foi apresentado pela primeira vez nas Comptes Rendus das sessões da Academia de Ciências dedicadas às memórias e comunicações dos membros e correspondentes da academia. Em razão dessa degenerescência adicional, não é de estranhar que as propriedades essenciais desses dois campos centrais tivessem sido estudadas por Newton. Newton refere-se ao oscilador harmônico isotrópico na proposição X do Livro I, e a lei do inverso do quadrado na proposição XI. ${ }^{4}$ Newton demonstra que ambos os campos dão origem a uma órbita elíptica com a diferença que, no primeiro caso, a força aponta para o centro geométrico da elipse e, no segundo, para um dos focos. 0 resultado de Bertrand, conhecido como teorema de Bertrand, continua a fascinar antigas e novas gerações de estudantes da mecânica newtoniana, e não deve ser surpresa saber que novos trabalhos sobre o tema continuam a aparecer na literatura. Como exemplo de demonstrações perturbativas, o leitor pode consultar Greenberg, 1966; Tikochinsky, 1988; Brown, 1978; Zarmi, 2002. Podemos também encontrar na literatura demonstrações semeIhantes àquela do trabalho original de Bertrand, como Greenberg, 1966; Arnold, 1974; Goldstein \& Poole \& Safko, 2002. ${ }^{5}$ A demonstração de Bertrand é ao mesmo tempo concisa, elegante e não-perturbativa, ao contrário do que poderíamos ser levados a crer em razão do número de demonstrações alternativas que apelam para técnicas perturbativas.

Recentemente, apresentamos um método para a determinação do ângulo apsidal a partir de um campo de força central arbitrário e mostramos sob quais condições esse ângulo independe da energia e do momento angular e leva a órbitas fechadas. ${ }^{6}$ Como consequência, reobtivemos o teorema de Bertrand. Dado 0 interesse e a relevância desse teorema, os autores concluíram ser pertinente a sua tradução para a nossa língua, na firme crença de que é importante para a nossa evolução cultural e científica a disponibilidade de versões em língua portuguesa de trabalhos e livros que são patrimônio da humanidade como um todo.

A sessão em que Bertrand apresentou sua memória foi realizada na segunda-feira, 20 de outubro de 1873, sob a presidência do sr. de Quatrefages. Na presente tradução, mantivemos a numeração original das equações. 0 leitor atento observará que a maior parte das equações não está numerada e que, entre a equação (4) e a equação (6), há várias equações, nenhuma delas numerada. Procuramos manter também, na medida do possível, a notação original.

\section{Mecânica analítica - Teorema relativo ao movimento de um ponto atraído para um centro fixo; pelo sr. J. Bertrand}

As órbitas planetárias são curvas fechadas; esta é a causa principal da estabilidade do nosso sistema, e essa circunstância importante resulta da lei da atração que, quaisquer que sejam as circunstâncias iniciais, faz mover segundo uma circunferência de uma elipse cada corpo celeste que não é expulso de nosso sistema. Até agora não foi notado que a lei de atração newtoniana é a única que preenche essa condição.

Entre as leis de atração que supõem a ação nula a uma distância infinita, aquela da natureza é a única segundo a qual um móvel lançado arbitrariamente, com uma velocidade inferior a certo limite e atraído em direção a um centro fixo descreve necessariamente em torno desse centro uma curva fechada. Todas as leis de atração permitem órbitas fechadas, mas a lei da natureza é aquela que as impõe.

Demonstra-se esse teorema da seguinte maneira: seja $\varphi$ (r) a atração exercida a uma distância r sobre a molécula considerada e dirigida em direção ao centro da atração que tomaremos como origem das coordenadas. Denotando por r e $\theta$ as duas coordenadas polares do móvel, temos, em virtude de uma fórmula bem conhecida,

e, fazendo $\frac{1}{r}=z$

$$
\varphi(r)=\frac{k^{2}}{r^{2}}\left(\frac{1}{r}+\frac{d^{2} \frac{1}{r}}{d \theta^{2}}\right)
$$




$$
\begin{gathered}
r^{2} \varphi(r)=\psi(z), \\
\frac{d^{2} z}{d \theta^{2}}+z-\frac{1}{k^{2}} \psi(z)=0,
\end{gathered}
$$

Multipliquemos os dois membros por $2 d z$ e integremos. Fazendo

$$
2 \int \psi(z) d z=\tilde{\omega}(z)
$$

teremos

$$
\left(\frac{d z}{d \theta}\right)^{2}+z^{2}-\frac{1}{k^{2}} \tilde{\omega}(z)-h=0,
$$

sendo $h$ uma constante.

Disso deduz-se que

$$
d \theta= \pm \frac{d z}{\sqrt{h+\frac{1}{k^{2}} \tilde{\omega}(z)-z^{2}}} .
$$

Se a curva representada pela equação que relaciona $z \operatorname{com} \theta$ é fechada, o valor de $z$ terá máximos e mínimos para os quais $d z / d \theta$ será nula, e os raios vetores correspondentes, normais à trajetória, serão para ela necessariamente eixos de simetria. Ora, quando uma curva admite dois eixos de simetria, a condição necessária e suficiente para que ela seja fechada é que o ângulo entre eles seja comensurável com $\pi$. Portanto, se $\alpha$ e $\beta$ representam um mínimo de $z$ e o máximo que o sucede, a condição exigida é expressa pela equação

$$
m \pi=\int_{\alpha}^{\beta} \frac{d z}{\sqrt{h+\frac{1}{k^{2}} \tilde{\omega}(z)-z^{2}}},
$$

onde $m$ denota um número comensurável. Essa equação deve ser válida quaisquer que sejam $h \mathrm{e} k \mathrm{e}$, consequentemente, os limites $\alpha$ e $\beta$ que deles dependem.

Tem-se

$$
\begin{aligned}
& h+\frac{1}{k^{2}} \tilde{\omega}(\alpha)-\alpha^{2}=0, \\
& h+\frac{1}{k^{2}} \tilde{\omega}(\beta)-\beta^{2}=0,
\end{aligned}
$$

consequentemente

$$
\begin{aligned}
\frac{1}{k^{2}} & =\frac{\beta^{2}-\alpha^{2}}{\tilde{\omega}(\beta)-\tilde{\omega}(\alpha)}, \\
h & =\frac{\alpha^{2} \tilde{\omega}(\beta)-\beta^{2} \tilde{\omega}(\alpha)}{\tilde{\omega}(\beta)-\tilde{\omega}(\alpha)},
\end{aligned}
$$


e a equação (3) torna-se

$$
m \pi=\int_{\alpha}^{\beta} \frac{d z \sqrt{\tilde{\omega}(\beta)-\tilde{\omega}(\alpha)}}{\sqrt{\alpha^{2} \tilde{\omega}(\beta)-\beta^{2} \tilde{\omega}(\alpha)+\left(\beta^{2}-\alpha^{2}\right) \tilde{\omega}(z)-z^{3}[\tilde{\omega}(\beta)-\tilde{\omega}(\alpha)]}} .
$$

A função $\tilde{\omega}(z)$ deve ser tal que essa equação seja válida para todos os valores de $\alpha$ e $\beta$. Além disso, o número comensurável $m$ deve ser constante, porque, se ele variar de uma órbita para outra, uma variação infinitamente pequena das condições iniciais implicaria uma variação finita do número e da disposição dos eixos de simetria da trajetória.

Suponhamos que $\alpha$ e $\beta$ difiram infinitesimalmente, ou seja,

$$
\beta=\alpha+u,
$$

ficando $z$ compreendido entre $\alpha$ e $\beta$, podemos fazer

$$
z=\alpha+\gamma,
$$

e $\gamma$ será, como $u$, infinitesimalmente pequeno. Desprezando os infinitamente pequenos de segunda ordem, teremos

$$
\sqrt{\tilde{\omega}(\beta)-\tilde{\omega}(\alpha)}=\sqrt{u \tilde{\omega}^{\prime}(\alpha)}
$$

Na expressão colocada sob o radical no denominador da integral (4), os infinitamente pequenos de primeira ordem reduzem-se a zero, e o mesmo acontece com aqueles de segunda; são os de terceira que é preciso manter e, desprezando os infinitamente pequenos de quarta ordem, tem-se

$$
\begin{aligned}
& \alpha^{2} \tilde{\omega}(\beta)-\beta^{2} \tilde{\omega}(\alpha)+\left(\beta^{2}-\alpha^{2}\right) \tilde{\omega}(z)-z^{3}[\tilde{\omega}(\beta)-\tilde{\omega}(\alpha)]= \\
& {\left[\tilde{\omega}^{\prime}(\alpha)-\alpha \tilde{\omega}^{\prime \prime}(\alpha)\right]\left(u^{2} \gamma-u \gamma^{2}\right) .}
\end{aligned}
$$

A equação (4) torna-se

$$
m \pi=\int_{\alpha}^{\beta} \frac{d \gamma \sqrt{\tilde{\omega}^{\prime}(\alpha)}}{\sqrt{\tilde{\omega}^{\prime}(\alpha)-\alpha \tilde{\omega}^{\prime \prime}(\alpha)} \sqrt{u \gamma-\gamma^{2}}},
$$

isto é, efetuando a integração e suprimindo os fatores comuns,

$$
m=\sqrt{\frac{\tilde{\omega}^{\prime}(\alpha)}{\tilde{\omega}^{\prime}(\alpha)-\alpha \tilde{\omega}^{\prime \prime}(\alpha)}}
$$

ou

$$
\left(1-m^{2}\right) \tilde{\omega}^{\prime}(\alpha)+m^{2} \tilde{\omega}^{\prime \prime}(\alpha)=0
$$


Deduz-se

$$
\begin{aligned}
& \tilde{\omega}^{\prime}(\alpha)=\frac{A}{\alpha^{1 / m^{2}-1}}, \\
& \tilde{\omega}(\alpha)=A \frac{\alpha^{2-1 / m^{2}}}{2-\frac{1}{m^{2}}}+B,
\end{aligned}
$$

$A$ e $B$ denotando constantes.

A partir das relações supostas entre as funções $\omega, \psi$ e $\varphi$ resulta

$$
\begin{aligned}
& \psi(z)=\frac{A}{2 z^{1 / m^{2}-1}}, \\
& \varphi(z)=\frac{A}{2} r^{1 / m^{2}-3} .
\end{aligned}
$$

Esta é a única lei de atração possível, $m$ aqui denotando um número comensurável qualquer; mas disto não resulta que ela preencha, qualquer que seja $m$, todas as condições do enunciado. De fato, deve-se ter para todos os valores de $\alpha$ e de $\beta{ }^{8}$

$$
m \pi=\int_{\alpha}^{\beta} \frac{d z \sqrt{\frac{1}{\beta^{1 / m^{2}-2}}-\frac{1}{\alpha^{1 / m^{2}-2}}}}{\sqrt{\frac{\alpha^{2}}{\beta^{1 / m^{2}-2}}-\frac{\beta^{2}}{\alpha^{1 / m^{2}-2}}+\left(\beta^{2}-\alpha^{2}\right) \frac{1}{z^{1 / m^{2}-2}}-z^{2}\left[\frac{1}{\beta^{1 / m^{2}-2}}-\frac{1}{\alpha^{1 / m^{2}-2}}\right]}}
$$

Suponhamos inicialmente $1 / m^{2}-2$ negativo; façamos $\alpha=0, \beta=1$, a equação torna-se

e a equação (6) fornece

$$
m \pi=\int_{0}^{1} \frac{d z}{\sqrt{\frac{1}{z^{1 / m^{2}-2}}-z^{2}}}=\int_{0}^{1} \frac{z^{1 /\left(2 m^{2}\right)-1} d z}{\sqrt{1-z^{1 / m^{2}}}}
$$

$$
\begin{gathered}
m \pi=m^{2} \pi, \\
m=1 .
\end{gathered}
$$

A lei de atração correspondente é:

$$
\varphi(r)=\frac{A}{r^{2}}
$$

Se supusermos $1 / m^{2}-2$ positivo, a equação (6) para $\alpha=1, \beta=0$, 


$$
m \pi=\int_{0}^{1} \frac{d z}{\sqrt{1-z^{2}}}=\frac{\pi}{2} .
$$

Disto deduz-se que $m=1 / 2$, e a lei de atração correspondente é

$$
\varphi(r)=A r .
$$

Portanto, somente duas leis preenchem as condições exigidas: a da natureza, pela qual a órbita fechada tem apenas um eixo de simetria passando pelo centro da ação; e a da atração proporcional à distancia, de acordo com a qual existem dois.

Nosso ilustre correspondente, sr. Chebychev, a quem comuniquei a demonstração anterior, judiciosamente me fez observar que o teorema, inútil hoje em dia para a teoria tão perfeita dos planetas, poderá ser invocada de modo útil para estender às estrelas duplas as leis da atração newtoniana.

\section{Notas e referências bibliográficas}

Filadelfo Cardoso Santos e Vitorvani Soares são professores adjuntos do Instituto de Física da UFRJ, e Alexandre Carlos Tort é professor associado da mesma instituição. Os autores agradecem ao prof. dr. Carlos Farina pela leitura atenta do manuscrito, seguida de críticas e sugestões.

E-mails: filadelf@if.ufrj.br, vsoares@if.ufrj.br, tort@if.ufrj.br.

1 Em nosso último acesso, em 08/09/2010, o trabalho original de Bertrand foi encontrado na biblioteca on-line Gallica no endereço: http://gallica.bnf. fr/ark:/12148/bpt6k3034n.image.F849.langFR.

2 BERTRAND, J. C. R. Acad. Sci. Paris 77, p. 849-853, 1873.

3 BERTRAND, J. Les fondateurs de l'astronomie moderne: Copernic, Tycho Brahé, Kepler, Gallilée, Newton. Paris: Hetzel, 1865; BERTRAND, J. Os fundadores da astronomia moderna. Rio de Janeiro: Contraponto, 2008.

4 NEWTON, I. Philosophiae naturalis principia mathematica. Tradução inglesa de A. Motte revista por F. Cajori. Berkeley, CA: University of California Press, 1962 [Londres: Royal Society, 1687].

5 GREENBERG, D. F. Am. J. Phys. 34, p. 1101-1109, 1966; TIKOCHINSKY, Y. Am. J. Phys. 56, p. 1073-1075, 1988; BROWN, L. S. Am. J. Phys. 46, p. 930-931, 1978; ZARMI, Y. Am. J. Phys. 70, p. 446-449, 2002; ARNOLD, V. I. Mathematical Methods of classical mechanics. Berlim: Springer, 1974; GOLDSTEIN, H.; POOLE, C.; SAFKO, J. Classical mechanics. Reading, MA: Addison-Wesley, 2002, cap. 3, sec. 3.6, p. 89-92.

6 SANTOS, F. C.; SOARES, V.; TORT, A.C. Physical Review E 79, 036605, 2009.

7 No original francês, molécule. Bertrand refere-se a uma partícula.

8 Mantivemos a numeração do original, no qual a numeração das equações salta de (4) para (6).

[ Artigo recebido em 07/2010 | Aceito em 01/2011 ] 